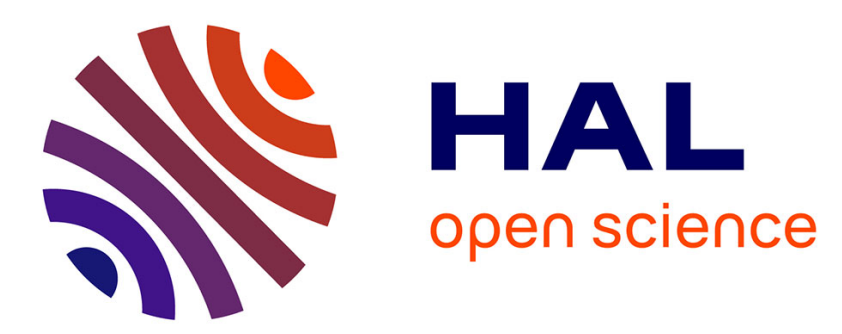

\title{
Effet du couplage thermomécanique sur la propagation d'un front de changement de phase
}

Xavier Balandraud, Sylvain Leclercq, Robert Peyroux, André Chrysochoos

\section{To cite this version:}

Xavier Balandraud, Sylvain Leclercq, Robert Peyroux, André Chrysochoos. Effet du couplage thermomécanique sur la propagation d'un front de changement de phase. Comptes rendus de l'Académie des sciences. Série IIb, Mécanique, 2001, 329 (8), pp.621-626. 10.1016/s1620-7742(01)01376-9 . hal03350332

\section{HAL Id: hal-03350332 \\ https://hal.science/hal-03350332}

Submitted on 21 Sep 2021

HAL is a multi-disciplinary open access archive for the deposit and dissemination of scientific research documents, whether they are published or not. The documents may come from teaching and research institutions in France or abroad, or from public or private research centers.
L'archive ouverte pluridisciplinaire HAL, est destinée au dépôt et à la diffusion de documents scientifiques de niveau recherche, publiés ou non, émanant des établissements d'enseignement et de recherche français ou étrangers, des laboratoires publics ou privés. 


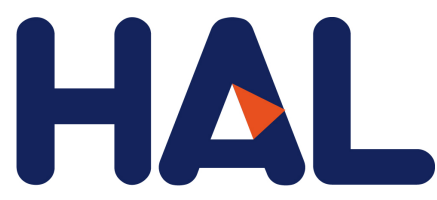

archives-ouvertes

\section{Effet du couplage thermomécanique sur la propagation d'un front de changement de phase}

Xavier Balandraud, Sylvain Leclercq, Robert Peyroux, André Chrysochoos

\section{To cite this version:}

Xavier Balandraud, Sylvain Leclercq, Robert Peyroux, André Chrysochoos. Effet du couplage thermomécanique sur la propagation d'un front de changement de phase. Comptes rendus de l'Académie des sciences. Série IIb, Mécanique, Elsevier, 2001, 329 (8), pp.621-626. 10.1016/s1620-7742(01)013769. hal-03350332

\section{HAL Id: hal-03350332 \\ https://hal.archives-ouvertes.fr/hal-03350332}

Submitted on 21 Sep 2021

HAL is a multi-disciplinary open access archive for the deposit and dissemination of scientific research documents, whether they are published or not. The documents may come from teaching and research institutions in France or abroad, or from public or private research centers.
L'archive ouverte pluridisciplinaire HAL, est destinée au dépôt et à la diffusion de documents scientifiques de niveau recherche, publiés ou non, émanant des établissements d'enseignement et de recherche français ou étrangers, des laboratoires publics ou privés. 


\title{
Effet du couplage thermomécanique sur la propagation d'un front de changement de phase
}

\author{
Xavier BALANDRAUD ${ }^{\text {a,b }}$, André CHRYSOCHOOS ${ }^{\text {a }}$, Sylvain LECLERCQ ${ }^{\text {a }}$, Robert PEYROUX ${ }^{\text {a }}$ \\ ${ }^{a}$ Laboratoire de mécanique et génie civil, Université Montpellier II, case 081, place E. Bataillon, \\ 34005 Montpellier cedex 5, France \\ ${ }^{b}$ Department of Mathematical Methods and Models for Scientific Applications, \\ Università degli Studi di Padova, Via Belzoni, 7, 35131 Padova, Italy \\ Courriel : balandr@dmsa.unipd.it; peyroux@Imgc.univ-montp2.fr
}

Résumé. Un système thermographique permet d'analyser les effets calorimétriques liés à la propagation d'un front de changement de phase dans un monocristal de CuZnAl. Sous charge constante, le déplacement du front induit un fluage de l'éprouvette dépendant fortement des conditions d'échange thermique avec le milieu extérieur. Le rôle clé du couplage thermomécanique peut être mis en évidence en inversant le flux de chaleur reçu par l'éprouvette : on observe alors une inversion du sens de propagation du front et une recouvrance de la déformation de fluage.

milieux continus / imagerie infrarouge / chaleur latente / front de changement de phase / localisation / alliages à mémoire de forme

\section{Influence of the thermomechanical coupling on the propagation of a phase change front}

\begin{abstract}
Calorimetric effects related to the propagation of a phase change front in a monocrystalline shape memory alloy $\mathrm{CuZnAl}$ were derived from thermographic data analysis. At constant load, the displacement of the front induces a creep of the sample strongly depending on thermal exchanges with the surroundings. The main role played by the thermomechanical couplings can be pointed out by reversing the heat flux at the boundary of the sample: this leads to an inversion of the front propagation way associated with a recovery of the creep strain.
\end{abstract}

continuum mechanics / imaging techniques / latent heat / phase change front /localization / shape memory alloys

\section{Abridged English version}

During tensile tests on monocrystalline shape memory alloys (SMA), strain induced by the propagation of phase change fronts has already been evidenced in [1-4]. In this work, an infrared image processing is used in order to analyse the calorimetric effects linked to the propagation of these fronts. The main purpose of this Note is to point out the importance of the thermomechanical couplings on the understanding of the material behavior when designing SMA structures. These couplings are linked to a phase transformation induced by mechanical and/or thermal loadings, and involve material/structure interactions due to the heat diffusion. 
Quasi-static tensile tests were performed at constant room temperature on a CuZnAl monocrystalline SMA initially in an austenitic state. Thermal response of the sample was monitored by an infrared camera. Derivation of heat sources which accompany the thermodynamic process was then done using the heat equation (equation (1)) and thermal data. Principles and methods of heat sources calculation were widely presented in [6,7]. For shape memory alloys, it is now established [8-10] that heat sources are essentially rates of latent heat per unit volume (equation (2)).

Figure $1 A$ presents a load-controlled test, divided in three parts: a loading stage $(a-b)$, a holding stage at a constant stress $(b-c)$, and a unloading stage $(c-d)$. The evolution of a temperature profile along one generatrix of the cylindric sample is presented in figure $1 B$. In the same way, the corresponding heat sources are plotted in figure 1C. The first part $\left(a-a^{\prime}\right)$ of the loading stage is associated with the elastic behavior of the material, involving small temperature variations, and slight deformation of the sample. At time $a^{\prime}$, strong and positive heat sources concentrate at the bottom of the sample, in a zone moving during the loading $\left(a^{\prime}-b\right)$. The displacement of this zone is associated with the propagation of a phase change front (austenite/martensite exothermal transformation). When the stress is held $(b-c)$, sources of lower intensity are observed, and the zone where they concentrate moves slower. The associated phase transformation involves also a localized strain and then a displacement of the cross-head (figure 1A). Finally, we observe during the unloading stage $(c-d)$ the propagation of a zone where negative heat sources are concentrated (martensite/austenite endothermal transformation).

For all the stages, and due to heat diffusion, phase change localization is easier to track from heat sources analysis (figure $1 C$ ) than from temperature fields (figure $1 B$ ). Indeed, the time evolution of temperature in one characteristic point ( figure $2 B$ ) is the resulting effect of all the heat transfer modes: conduction of the latent heat within the sample, and convection, radiation and conduction of heat between the sample and the surroundings. Nervertheless, analysis of heterogeneous temperature profiles is consistent with the existence of a wave-like front moving along the sample (figure $2 A$ ). Indeed, the maximun amplitude of temperature variations may be roughly associated with the current position of the front.

The propagation of the phase change front is tightly related to the thermomechanical couplings. Due to these couplings, the mechanical behavior of SMA is strongly dependent on the temperature and consequently on the thermal boundary conditions. Figure 3 shows two mechanical responses obtained with two different values of the heat flux exchanged between the sample and the surroundings. The creep of the sample, observed when the stress is kept constant, is induced by the propagation of the front, the direction of which is related to heat exchanges. The higher the value of heat flux at the boudary of the sample is, the faster the front propagates. As an evidence of the existence of such mechanisms, one can obtain up to an inversion of phase change way by reversing the sign of the exchanged heat flux (figure 4).

\section{Introduction}

Lors d'essais de traction sur des éprouvettes monocristallines en alliages à mémoire de forme, les effets cinématiques liés à la propagation de fronts de changement de phase austénite-martensite ont été mis en évidence par de nombreux auteurs [1-4]. Nous proposons ici d'utiliser un traitement numérique d'images infrarouges pour analyser les effets calorimétriques dus à la propagation de ces fronts de changement de phase lors de chargements mécaniques et thermiques. L'objectif de cette Note est de montrer l'importance des mécanismes de couplage thermomécanique dans la compréhension du comportement d'un matériau dont la réponse mécanique est fortement dépendante de la température, et des effets de structure induits par la diffusion de la chaleur au sein de l'éprouvette. 


\section{Calcul des sources de chaleur}

Le cadre thermodynamique utilisé est celui des Matériaux Standards Généralisés [5]. Les variables d'état choisies sont la température $T$, la déformation $\epsilon$ et la fraction volumique de martensite $x$. En notant $C$ la capacité calorique (à $\epsilon$ et $x$ constants), $k$ le coefficient de conduction isotrope, $d_{1}$ la dissipation intrinsèque et $\psi$ le potentiel énergie libre, l'équation de la chaleur s'écrit, en négligeant les termes de convection :

$$
\rho C \frac{\partial T}{\partial t}-k \Delta T=d_{1}+\rho T \frac{\partial^{2} \psi}{\partial T \partial \epsilon} \dot{\epsilon}+\rho T \frac{\partial^{2} \psi}{\partial T \partial x} \dot{x}=w_{\mathrm{ch}}^{\prime}
$$

Les méthodes permettant de passer des températures aux sources de chaleur sont largement détaillées dans [6,7]. Le traitement d'images consiste, entre autres, à estimer le membre de gauche de l'équation (1) à partir des cartes de températures enregistrées par la caméra. Lors d'une transformation pseudoélastique d'un AMF, la source de chaleur $w_{\mathrm{ch}}^{\prime}$ peut être assimilée au taux de chaleur latente de changement de phase, comme cela a été montré dans [8-10]. Dans ce qui suit, nous avons utilisé une équation de la chaleur moyennée sur chaque section droite de l'éprouvette cylindrique d'axe $z$ :

$$
\rho C\left(\frac{\partial \theta}{\partial t}+\frac{\theta}{\tau_{\mathrm{th}}}\right)-k \frac{\partial^{2} \theta}{\partial z^{2}} \approx \rho T \frac{\partial^{2} \psi}{\partial T \partial x} \dot{x}
$$

où $\theta$ est la variation de température moyenne de la section par rapport à la température d'équilibre, et où $\tau_{\text {th }}$ est une constante de temps caractéristique des fuites thermiques radiales.

\section{Description des expériences}

Des essais quasi-statiques de traction sont réalisés à température ambiante constante sur une éprouvette cylindrique de diamètre $3,85 \mathrm{~mm}$ et de longueur utile $24 \mathrm{~mm}$. L'AMF choisi est un monocristal d'alliage à mémoire de forme $\mathrm{Cu}_{70} \mathrm{Zn}_{25} \mathrm{Al}_{4}(\mathrm{pd} \%)$ [11,12]. Les principales caractéristiques thermomécaniques de cet alliage sont données dans le tableau ci-dessous (caractéristiques détaillées dans [9]). La température ambiante est telle que l'éprouvette est à l'état austénitique en début d'essai.

Le chargement mécanique de l'essai présenté sur la figure $1 A$ est le suivant :

- Etape $a-b$ : Mise en charge de l'éprouvette à force controlée $\left(\dot{F}=93 \mathrm{~N} \cdot \mathrm{s}^{-1}\right)$.

- Etape $b-c$ : Maintien de la force à $885 \mathrm{~N}$ pendant 2 minutes.

- Etape $c-d$ : Retour à force nulle $\left(F=-93 \mathrm{~N} \cdot \mathrm{s}^{-1}\right)$.

La figure $1 B$ montre l'évolution dans le temps du profil de température $\theta$ enregistré sur une génératrice de l'éprouvette. L'axe des abscisses représente le temps gradué en numéro d'image, la fréquence d'acquisition étant constante et de l'ordre de 2 images par seconde. L'axe des ordonnées représente la génératrice de l'éprouvette. Dans le même esprit, la figure $1 C$ présente l'évolution des sources de chaleur sur cette même génératrice.

La partie $\left(a-a^{\prime}\right)$ de la charge correspond à la réponse élastique de la structure, se traduisant par de faibles variations de température et un faible déplacement de la traverse. En $a^{\prime}$, le domaine de transition est atteint dans une zone située au bas de l'éprouvette. Les sources de chaleur positives se concentrent alors dans cette zone qui se déplace régulièrement le long de l'éprouvette durant le chargement $\left(a^{\prime}-b\right)$. Ce déplacement est

Tableau. Caractéristiques générales du matériau utilisé, autour de $30^{\circ} \mathrm{C}$.

\begin{tabular}{lccccc}
\hline $\begin{array}{c}\text { Masse } \\
\text { volumique } \\
\mathrm{Kg} \cdot \mathrm{m}^{-3}\end{array}$ & $\begin{array}{c}\text { Capacité } \\
\text { calorifique } \\
\mathrm{J} \cdot \mathrm{Kg}^{-1} \cdot \mathrm{K}^{-1}\end{array}$ & $\begin{array}{c}\text { Conductivité } \\
\text { thermique } \\
\mathrm{W} \cdot \mathrm{m}^{-1} \cdot \mathrm{K}^{-1}\end{array}$ & $\begin{array}{c}\text { Dilatation } \\
\text { thermique } \\
\mathrm{K}^{-1}\end{array}$ & $\begin{array}{c}\text { Chaleur } \\
\text { latente } \\
\mathrm{J} \cdot \mathrm{Kg}^{-1} \cdot{ }^{\circ} \mathrm{C}\end{array}$ & $\begin{array}{c}\text { Températures } \\
\text { de transition } \\
{ }^{\circ} \mathrm{C}\end{array}$ \\
\hline$\rho=7800$ & $C=390$ & $\lambda=120$ & $\alpha=1,9 \times 10^{-5}$ & $L=7000$ & $M s=23 \quad M f=12$ \\
& & & & $A s=22 \quad A f=29$ \\
\hline
\end{tabular}




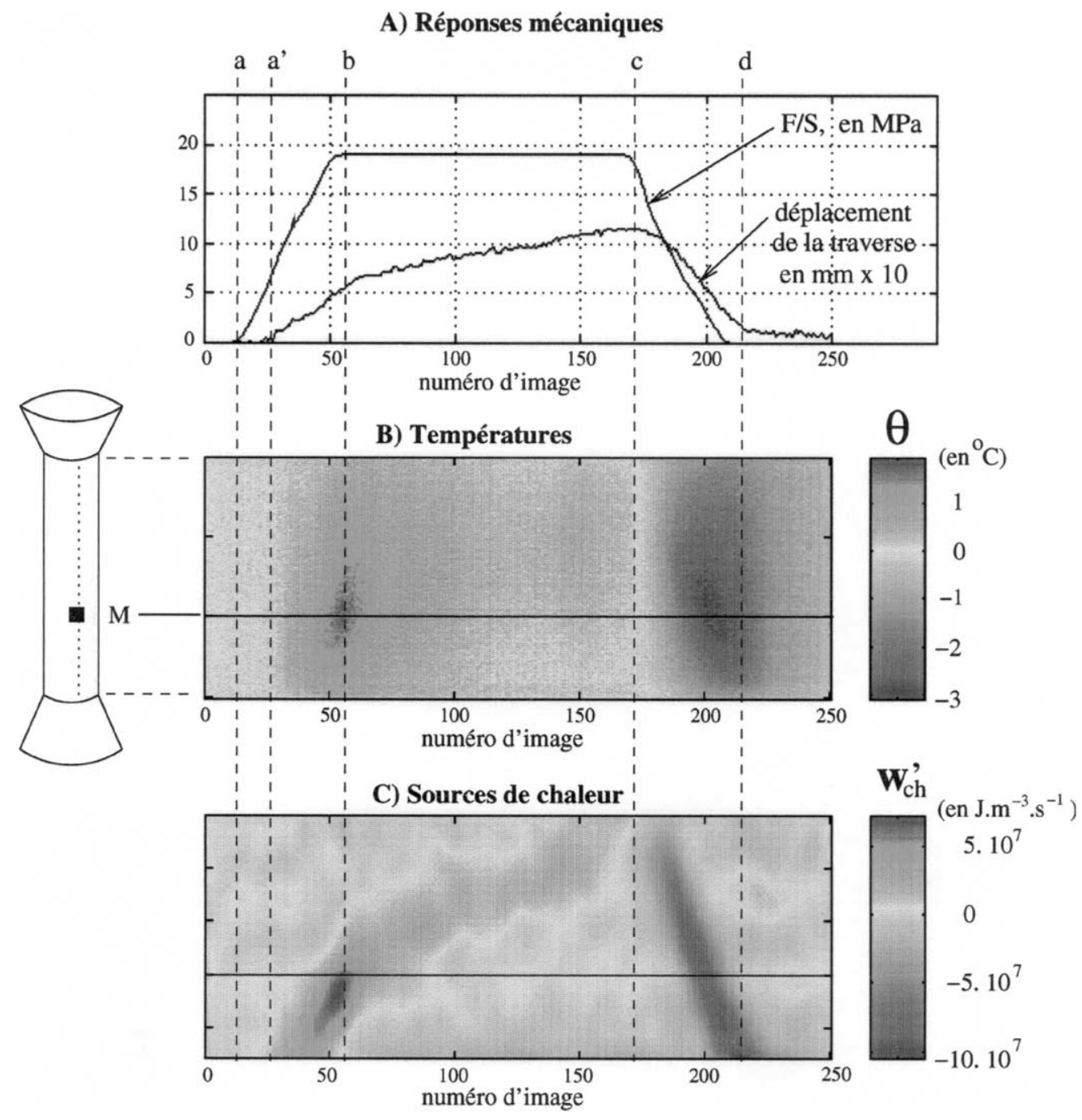

Figure 1. A) Force et déplacement de la traverse en fonction du temps. B) Variations de température $\theta$ en fonction du temps, le long d'une génératrice de l'éprouvette. C) Sources de chaleur $w_{\mathrm{ch}}^{\prime}$ en fonction du temps, le long d'une génératrice de l'éprouvette.

Figure 1. A) Time evolution of stress and cross-head displacement. B) Time evolution of temperature variations $\theta$ along the sample. $C$ ) Time evolution of heat sources $w_{\mathrm{ch}}^{\prime}$ along the sample.

associé à la propagation d'un front de changement de phase exothermique traduisant localement le passage d'un état austénitique à un état martensitique.

Durant l'étape où la charge est maintenue constante $(b-c)$, les sources ont une intensité moindre et le front de changement de phase se déplace plus lentement. Ce déplacement génère donc localement des déformations et globalement un fluage de l'éprouvette (figure 1A).

Enfin, durant la décharge $(c-d)$, on assiste à la propagation d'un front de sources de chaleur négatives dues à une transformation martensite/austénite endothermique.

Notons qu'en terme de température (figure $1 B$ ), l'échauffement $\left(a^{\prime}-b\right)$ et le refroidissement $(c-d)$ de l'éprouvette sont nettement observables, alors que la propagation du front est plus difficile à discerner 

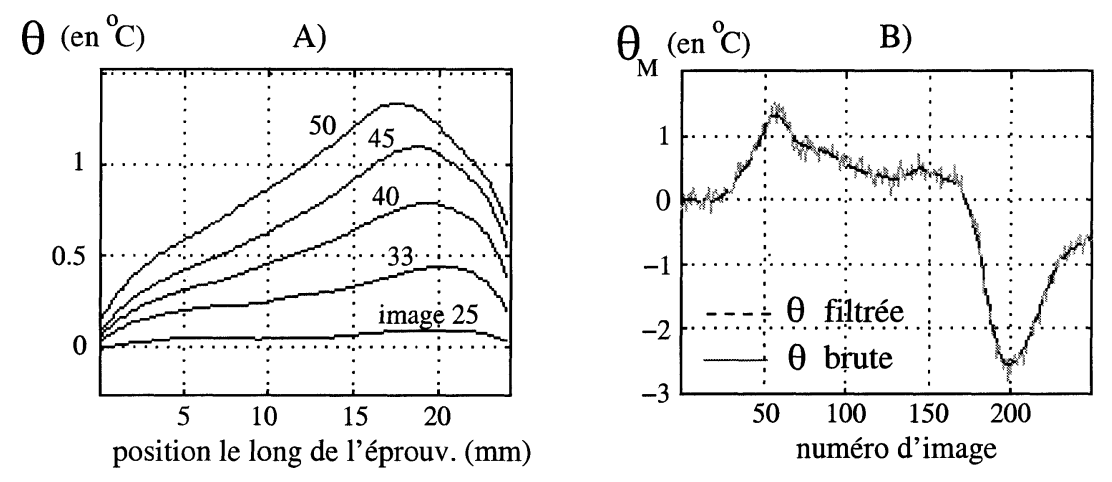

Figure 2. A) Profils longitudinaux de température à différents instants. B) Température au point $\mathrm{M}$ au cours de l'essai.

Figure 2. A) Longitudinal temperature profiles at different times. B) Temperature evolution at point $M$ throughout the test.

durant le fluage $(b-c)$ du fait de la diffusion de la chaleur. En terme de source de chaleur (figure 1C), la localisation du changement de phase apparaît distinctement tout au long de l'essai.

La figure $2 A$ montre cinq profils de température dans l'intervalle $\left(a^{\prime}-b\right)$. Ils permettent de visualiser l'hétérogénéité du champ de température liée à la propagation du front et aux fuites thermiques, la température maximale pour chaque profil étant approximativement associée à la position courante de ce front.

La figure $2 B$ présente l'évolution de la température au cours du temps en un point $\mathrm{M}$ de l'éprouvette. Durant l'étape où la force est maintenue constante $(b-c)$, cette température ne revient pas à la valeur ambiante. Deux mécanismes régissent son évolution : un échauffement par conduction provenant du front de changement de phase, et un refroidissement dû aux fuites de chaleur vers le milieu extérieur.

\section{Discussion}

Les effets calorimétriques associés à la transformation de phase sous contrainte induisent des variations de température importantes qui influent sur la cinétique de changement de phase. En effet, les variations maximales de température durant l'essai atteignent cinq degrés, et sont à comparer aux onze degrés caractérisant la largeur du domaine de transition [9]. La réponse mécanique locale dépend donc de la température, qui est elle-même fonction du taux de chaleur latente et des échanges thermiques dans la structure et entre la structure et l'extérieur (équation (2)). Lorsque le chargement mécanique est maintenu constant, la vitesse de propagation du front est alors directement liée aux conditions d'échange thermique entre l'éprouvette et le milieu extérieur. On obtient donc une déformation de l'éprouvette à contrainte constante, qui traduit une dépendance du comportement par rapport au temps et provoque le fluage de l'éprouvette, gouverné ici par la diffusion thermique. Pour s'en convaincre, nous avons augmenté les coefficients d'échange par convection dans l'enceinte thermique et obtenu des vitesses de propagation de front supérieures (figure 3). L'exemple le plus significatif est celui pour lequel le flux de chaleur échangé $\Phi$ a été inversé en élevant brusquement la température de l'air ambiant. On observe alors une inversion immédiate du changement de phase entraînant une recouvrance sous contrainte de la déformation de l'éprouvette («fluage inverse») (figure 4).

Ces phénomènes mécaniques remarquables proviennent du couplage thermomécanique qui traduit le changement de phase. Il confère au matériau un comportement dépendant du temps via la diffusion de la chaleur. Lorsque la transformation de phase est localisée, comme ici, dans un front, l'hétérogénéité du champ de sources de chaleur renforce l'importance des fuites thermiques et amplifie l'interaction matériaustructure. 
$\mathrm{F} / \mathrm{S}$ (en MPa)

A)

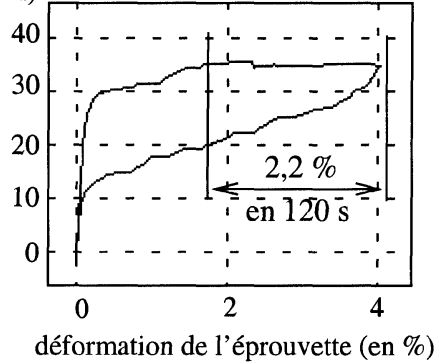

$\mathrm{F} / \mathrm{S}$ (en MPa)

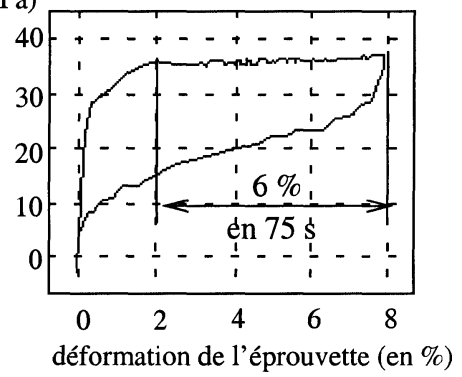

Figure 3. Réponses mécaniques de l'éprouvette pour deux valeurs du coefficient d'échange thermique $\left(h_{A}<h_{B}\right)$.

Figure 3. Mechanical responses of the sample for two different values of the heat exchange coefficient $\left(h_{A}<h_{B}\right)$.

$\mathrm{F} / \mathrm{S}$

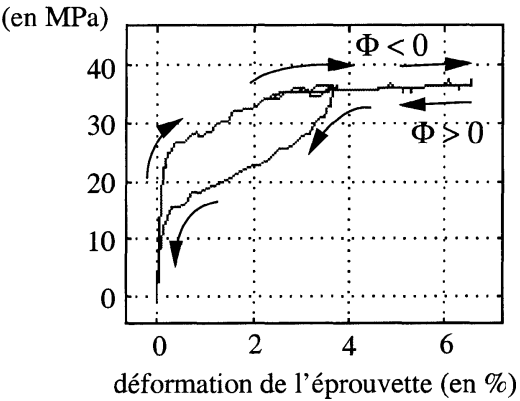

Figure 4. Fluage et fluage inverse lors d'une inversion du flux de chaleur échangé $\Phi$.

Figure 4. Creep and reverse creep due to heat flux $\Phi$ inversion.

\section{Références bibliographiques}

[1] Shaw J.A., Kyriakides S., Thermomechanical aspects of NiTi, J. Mech. Phys. Solids 43 (1995) 1243-1281.

[2] Jesse Lim T., McDowell D.L., Mechanical behavior of a Ni-Ti shape memory alloy under axial-torsional proportional and nonproportional loading, J. Eng. Mater. Technol. 121 (1999) 9-18.

[3] Orgeas L., Favier D., Stress-induced martensitic transformation of a NiTi alloy in isothermal shear, tension and compression, Acta Mater. 46 (1998) 5579-5591.

[4] Liu Yi., Liu Yo., van Humbeeck J., Luders-like deformation associated with martensite reorientation in NiTi, Scripta Materialia 39 (1998) 1047-1055.

[5] Halphen B., Nguyen Q.S., Sur les matériaux standards généralisés, J. Mécanique 14 (1975) 39-63.

[6] Louche H., Analyse par thermographie infrarouge des effets dissipatifs de la localisation dans les aciers, Thèse, Montpellier, 1999.

[7] Chrysochoos A., Louche H., An infrared image processing to analyse the calorific effects accompanying strain localisation, Int. J. Engng. Sci. 38 (2000) 1759-1788.

[8] Chrysochoos A., Löbel M., Maisonneuve O., Couplages thermomécaniques du comportement pseudoélastique d'alliages Cu-Zn-Al et Ni-Ti, C. R. Acad. Sci. Paris, Série IIb 320 (1995) 217-223.

[9] Tuffraud-Löbel M., Caractérisation thermomécanique d'alliages à mémoire de forme de type NiTi et $\mathrm{CuZnAl} \mathrm{;}$ domaine de transition et cinétique de changement de phase, Thèse, Montpellier, 1995.

[10] Peyroux R., Chrysochoos A., Licht C., Löbel M., Thermomechanical couplings and pseudoelasticity of shape memory alloys, Int. J. Engng. Sci. 36 (1998) 489-509.

[11] Balandraud X., Changement de phase et changements d'échelle dans des alliages à mémoire de forme, Thèse, Montpellier, 2000.

[12] Leclercq S., Analyse thermomécanique de la transformation martensitique dans les alliages à mémoire de forme par mesure acoustique, microscopie optique et thermographie infrarouge, Thèse, Montpellier, 2001. 